\title{
Juiz Online no ensino de Programação Introdutória - Uma Revisão Sistemática da Literatura
}

\author{
Rodrigo Elias Francisco $^{1}$, Cleon X. Pereira Júnior ${ }^{2}$, Ana Paula Ambrósio ${ }^{3}$ \\ ${ }^{1}$ Instituto Federal Goiano (IFGoiano) \\ Morrinhos - GO - Brasil \\ ${ }^{2}$ Instituto Federal Goiano (IFGoiano) \\ Campos Belos - GO - Brasil \\ ${ }^{3}$ Instituto de Informática - Universidade Federal de Goiás (UFG) \\ Goiânia - GO - Brasil \\ \{rodrigo.francisco,cleon.junior\}@ifgoiano.edu.br, apaula@inf.ufg.br
}

\begin{abstract}
The use of Online Judge systems in computer education can automate student feedback and make teacher work more productive. This article presents a Systematic Review of the Literature (RSL) that contributes to the specification of an Online Judge for use in introductory programming education (CS1). The RSL synthesis is subdivided into: benefits, problems, functional requirements and non-functional requirements. The results indicate aspects that must be taken into account when chosing an Online Judge for CS1, as well as a list of tools and how they treat these aspects. Critical issues related to the validation of tools were observed.
\end{abstract}

Resumo. O uso de sistemas de Juiz Online no ensino de computação pode automatizar o feedback oferecido a aluno e tornar o trabalho do professor mais produtivo. Este artigo apresenta uma Revisão Sistemática da Literatura (RSL) que contribui para a especificação de um Juiz Online a ser usado no ensino de programação introdutória (CS1). Para isso, a síntese da RSL se subdividiu em: benefícios, problemas, requisitos funcionais e requisitos não-funcionais. Os resultados apontam para aspectos que devem ser levados em consideração na escolha de um Juiz Online para CS1, assim como uma lista de ferramentas e como estas tratam os aspectos levantados. Observou-se que a seleção das ferramentas ainda apresenta questões críticas relacionadas à validação das mesmas.

\section{Introdução}

As disciplinas de Introdução à Programação de Computadores, conhecidas na literatura como Computer Science 1 (CS1), são objetos de pesquisas científicas dada a dificuldade que muitos alunos sentem na elaboração de algoritmos e implementação dos mesmos usando uma linguagem de programação. Programar exige elevado nível de generalização, abstração, pensamento crítico [Gomes et al. 2008] e a presença de modelos mentais adequados [Byrne and Lyons 2001, Wilson and Shrock 2001]. Outros fatores, como motivação [Rountree et al. 2004], também têm impacto na aprendizagem de CS1.

Apesar de não haver um consenso quanto à melhor metodologia ou linguagem de programação a ser usada, observa-se que a disciplina de CS1 necessita de que o aluno resolva uma grande quantidade de problemas. No entanto, em uma turma heterogênea, $o$ planejamento e correção de problemas gera uma sobrecarga no professor, dificultando o feedback aos alunos, o que pode causar frustrações no processo de ensino-aprendizagem 
V Congresso Brasileiro de Informática na Educação (CBIE 2016)

Anais do XXVII Simpósio Brasileiro de Informática na Educação (SBIE 2016)

[Vier et al. 2015]. Ferramentas que auxiliem neste processo têm sido propostas para preencher essa lacuna, sendo que uma das estratégias mais promissoras são os Juízes Online.

Um Juiz Online, também chamado de sistema de avaliação automática, oferece problemas para serem resolvidos com a submissão de códigos-fonte, em uma linguagem de programação, e os corrigem automaticamente [Kurnia et al. 2001] usando casos de teste. Cada caso de teste possui um conjunto de entradas e suas respectivas saídas. Para verificar se a resposta de um problema é uma solução correta, um programa passa por seu respectivo conjunto de testes. Esses sistemas são muito utilizados em competições de programação, como a Maratona de Programação organizada pela Sociedade Brasileira de Computação (SBC), e vêm sendo gradativamente adaptados para o ensino de CS1 devido à funcionalidade de automatizarem a correção dos programas.

Este artigo visa apresentar uma RSL sobre o uso de sistemas de Juiz Online no ensino de CS1, buscando contribuições à pergunta "Qual é a especificação mais adequada para que um juiz online atenda à disciplina de CS1?". Houve um planejamento com foco em mostrar os benefícios, os problemas, os requisitos funcionais e os requisitos não-funcionais sintetizados a partir dos trabalhos lidos, pois entende-se que diferentes contextos de ensino podem exigir diferentes estratégias educacionais.

\section{Metodologia da revisão}

Esta RSL segue as instruções elaboradas por [Kitchenham 2004], cuja condução se subdivide-se em três etapas: Planejamento, Execução e Resultados (Figura 1).

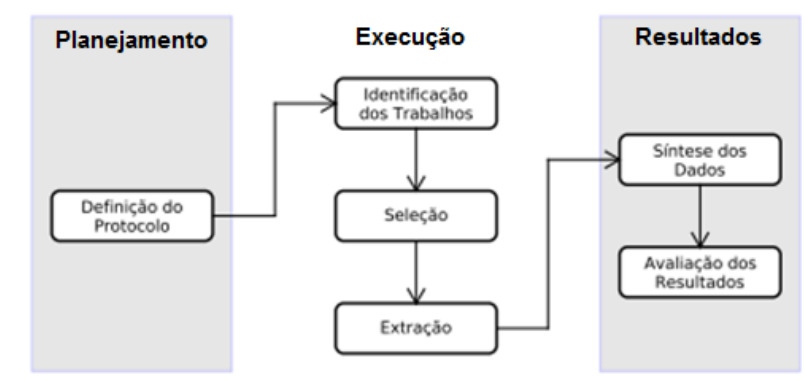

Figura 1. Fases de uma RSL - adaptado de [Vieira 2016]

\subsection{Planejamento}

Esta pesquisa tem como objetivo para responder à questão: 'Qual é a especificação mais adequada para que um Juiz Online atenda à disciplina de CS1?'. Para isso, outras quatro questões de pesquisa foram definidas:

- QP1: Quais os benefícios ao usar Juiz Online no ensino de CS1?

- QP2: Quais os problemas ao usar Juiz Online no ensino de CS1?

- QP3: Quais requisitos funcionais um Juiz Online precisa atender para ser usado no ensino de CS1?

- QP4: Quais requisitos não-funcionais um Juiz Online precisa atender para ser usado no ensino de CS1?

Para responder às perguntas levantadas, foi realizada uma busca nas bases de artigos científicos, considerando os trabalhos de 2010 a 2016 (Com a data limite de 
V Congresso Brasileiro de Informática na Educação (CBIE 2016)

Anais do XXVII Simpósio Brasileiro de Informática na Educação (SBIE 2016)

14/02/2016). Foram selecionadas as bases de pesquisa: IEEEXplorer Digital Library, ACM Digital Library, Springer, Science Direct e Scopus, que disponibilizam mecanismo de consulta via web, estão relacionadas a temas de Computação e Informática e também permitem filtro por ano de publicação. Foram considerados trabalhos publicados em português e inglês.

Tabela 1. Strings de busca para a seleção de artigos

\begin{tabular}{|c|l|}
\hline \multicolumn{2}{|c|}{ STRINGS DE BUSCA } \\
\hline Idioma & \multicolumn{1}{c|}{ String } \\
\hline Português & $\begin{array}{l}\text { ("juiz online"OR "avaliação automática"OR "correção automática") AND ("programação } \\
\text { introdutória"OR "introdução a programação"OR "ciência da computação 1"OR "CS1") }\end{array}$ \\
\hline Inglês & $\begin{array}{l}\text { ("online judge"OR "automated assessment"OR "automatic assessment") AND ("introductory } \\
\text { programming"OR "introduction to programming",OR "computer science 1",OR "CS1") }\end{array}$ \\
\hline
\end{tabular}

A tabela 2 apresenta os critérios de inclusão e exclusão. Como a pesquisa tem foco em abordar Juiz Online no ensino de CS1, os critérios de inclusão foram baseados em benefícios, problemas e os requisitos que devem ter um Juiz Online para ser utilizado no ensino de CS1.

Tabela 2. Critérios de Inclusão e Exclusão

\begin{tabular}{|l|l|l|}
\hline Critérios & ID & Descrição \\
\hline \multirow{4}{*}{ Exclusão } & E1 & Texto completo não disponível para acesso na Web \\
\cline { 2 - 3 } & E2 & Escrito em outro idioma \\
\cline { 2 - 3 } & E3 & Trabalho não aborda Juiz Online e ensino de CS1 \\
\cline { 2 - 3 } & E4 & Não é um capítulo de livro com resumo ou artigo de periódico ou de conferência \\
\hline \multirow{4}{*}{ Inclusão } & I1 & Aborda os benefícios ao usar Juiz Online no ensino de CS1 \\
\cline { 2 - 3 } & I2 & Aborda os problemas ao usar Juiz Online no ensino de CS1 \\
\cline { 2 - 3 } & I3 & Aborda os requisitos funcionais que um Juiz Online precisa atender para ser usado no ensino de CS1 \\
\cline { 2 - 3 } & I4 & Aborda os requisitos não-funcionais que um Juiz Online precisa atender para ser usado no ensino de CS1 \\
\hline
\end{tabular}

\subsection{Execução}

Um total de 40 trabalhos foram identificados, que seguem distribuições por ano e tipo de publicação conforme a Figura 2.
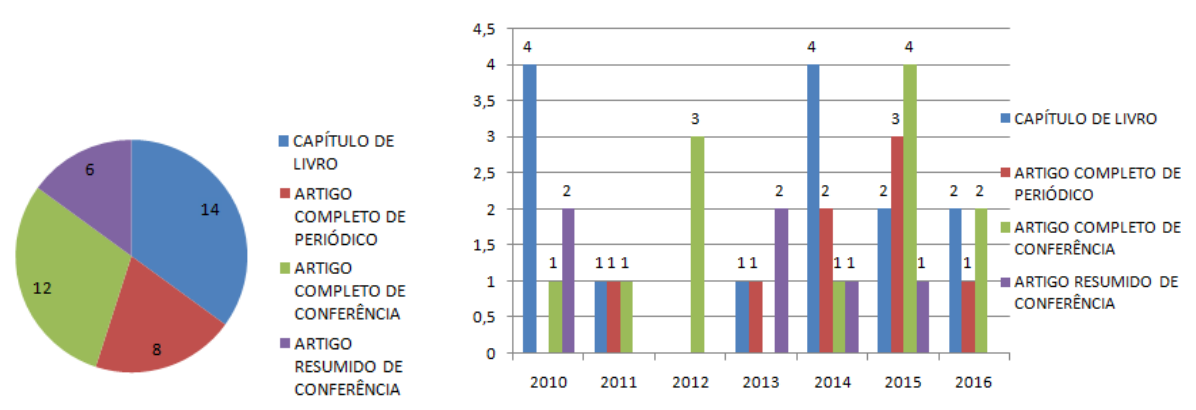

Figura 2. Número de Trabalhos identificados agrupados por tipo de publicação e por ano e tipo de publicação

Os dados mostram que as pesquisas sobre o assunto estão se popularizando. 2015 foi o ano em que houve o maior número de publicações, totalizando 10. Durante os dois primeiros meses de 2016, incluídos na pesquisa, de 01/01/2016 até 14/02/2016, ocorreram 5 publicações sobre o assunto. Isso indica um aumento no interesse pelo assunto a partir de 2015. 
V Congresso Brasileiro de Informática na Educação (CBIE 2016)

Anais do XXVII Simpósio Brasileiro de Informática na Educação (SBIE 2016)

A seleção preliminar dos trabalhos contou com a leitura do título, palavras-chave e resumo de cada trabalho. Dos artigos recuperados, 26 foram excluídos por não estarem ligados ao tema (E3) e 2 por não estarem disponíveis (E1). Os 12 trabalhos restantes passaram pela fase de extração, e foram usados para responder as questões de pesquisa, através da leitura completa dos trabalhos (Tabela 3).

Tabela 3. Trabalhos incluídos na extração conforme critérios de inclusão

\begin{tabular}{|c|c|c|}
\hline CRITÉRIO & REFERÊNCIAS & $\mathrm{N}^{\circ}$ TRABALHOS \\
\hline I1 & $\begin{array}{lll}\text { [Wang et al. 2011] } & \text { [AlShamsi and Elnagar 2011] [Pettit et al. 2015b] } \\
\text { [Rajala et al. 2016] } & \text { [Llana et al. 2012] [Georgouli and Guerreiro 2010] }\end{array}$ & 10 \\
\hline I2 & $\begin{array}{l}\text { [Petit et al. 2012] [Souza et al. 2015] [Vihavainen et al. 2013] [Sun et al. 2014] } \\
\text { [Wang et al. 2011] [AlShamsi and Elnagar 2011] [Pettit et al. 2015a] } \\
\text { [Pettit et al. 2015b] [Llana et al. 2012] [Georgouli and Guerreiro 2010] } \\
\text { [Pozenel et al. 2015] [Petit et al. 2012] [Souza et al. 2015] } \\
\text { [Vihavainen et al. 2013] [Sun et al. 2014] }\end{array}$ & 11 \\
\hline I3 & $\begin{array}{l}\text { TWang et al. 2011] [AlShamsi and Elnagar 2011] } \\
\text { [Rettit et al. 2015a] } \\
\text { [Rajala et al. 2016] [Llana et al. 2012] [Georgouli and Guerreiro 2010] } \\
\text { [Vihenel et al. 2015] [Petit et al. 2012] [Souza et al. 2015] }\end{array}$ & 11 \\
\hline I4 & $\begin{array}{l}\text { Rajala et al. 2016] [Clana et al. 2012] [Georgouli and Guerreiro 2010] } \\
\text { [Pozenel et al. 2015] [Petit et al. 2012] [Vihavainen et al. 2013] } \\
\text { [Sun et al. 2014] }\end{array}$ & 7 \\
\hline
\end{tabular}

\section{Resultados}

\section{QP1: Quais os benefícios ao usar Juiz Online no ensino de CS1?}

A Tabela 4 apresenta um resumo dos benefícios encontrados nos artigos selecionados. Existe um consenso de que o uso das ferramentas ajuda no ensino de CS1 por oferecer um ambiente que permite ao aluno regular sua aprendizagem, aumentando sua motivação, e, ao mesmo tempo, reduz a carga de trabalho do professor, que tem a sua disposição um conjunto de problemas já elaborados e testados e a possibilidade de automatizar a detecção de plágio e a emissão de relatórios.

Tabela 4. Benefícios encontrados nos trabalhos

\begin{tabular}{|c|c|c|}
\hline BENEFÍCIO & REFERÊNCIAS & No TRABALHOS \\
\hline $\begin{array}{l}\text { A - Existência de sistemas que são úteis } \\
\text { para o ensino }\end{array}$ & $\begin{array}{c}\text { [Pettit et al. 2015b], [Souza et al. 2015] } \\
\text { [Vihavainen et al. 2013], [Petit et al. 2012], } \\
\text { [Llana et al. 2012], [Sun et al. 2014] }\end{array}$ & 6 \\
\hline $\begin{array}{c}\text { B - Redução da carga de trabalho do } \\
\text { professor }\end{array}$ & $\begin{array}{c}\text { [Rajala et al. 2016], [Wang et al. 2011], } \\
\text { [AlShamsi and Elnagar 2011] }\end{array}$ & 3 \\
\hline C - Oferecer lista de exercícios extensa & [Petit et al. 2012] & 1 \\
\hline $\begin{array}{l}\text { D - Preocupações pedagógicas na } \\
\text { criação dos problemas }\end{array}$ & $\begin{array}{c}\text { [Llana et al. 2012], [Sun et al. 2014], } \\
\text { [Souza et al. 2015] }\end{array}$ & 3 \\
\hline E - Reduzir a ocorrência de plágio & [Rajala et al. 2016] & 1 \\
\hline F - Aprendizagem no ritmo do aluno & [Llana et al. 2012] & 1 \\
\hline G - Auto-aprendizagem & [Wang et al. 2011] & 1 \\
\hline H - Motivação & [Georgouli and Guerreiro 2010] & 1 \\
\hline I - Melhorar a estratégia pedagógica & $\begin{array}{c}\text { [Rajala et al. 2016], } \\
\text { [Vihavainen et al. 2013] }\end{array}$ & 2 \\
\hline $\begin{array}{c}\mathrm{J} \text { - Oferecer uma visão global e } \\
\text { temporal do desenvolvimento do aluno }\end{array}$ & [AlShamsi and Elnagar 2011] & 1 \\
\hline $\mathrm{K}$ - Assiduidade do aluno & [Georgouli and Guerreiro 2010] & 1 \\
\hline L - Transparência na correção & [Georgouli and Guerreiro 2010] & 1 \\
\hline
\end{tabular}

A Tabela 5 relaciona os sistemas de Juiz Online mencionados nos artigos com os benefícios identificados na Tabela 4. A descrição *Experiência UL-FCIS foi incluída pelo fato do artigo não apresentar o nome do sistema, visto que o que aborda são as ideias de um protótipo produzido e os relatos da experiência. 
V Congresso Brasileiro de Informática na Educação (CBIE 2016)

Anais do XXVII Simpósio Brasileiro de Informática na Educação (SBIE 2016)

Tabela 5. Juízes Online relacionados aos benefícios

\begin{tabular}{|l|c|c|c|c|c|c|c|c|c|c|c|c|}
\hline SISTEMA & A & B & C & D & E & F & G & H & I & J & K & L \\
\hline Mooshak [Georgouli and Guerreiro 2010] & & & & & & & & X & & & $\mathrm{X}$ & $\mathrm{X}$ \\
\hline AutoLEP [Wang et al. 2011] & & $\mathrm{X}$ & & & & & $\mathrm{X}$ & & & & & \\
\hline eGrader [AlShamsi and Elnagar 2011] & & $\mathrm{X}$ & & & & & & & & $\mathrm{X}$ & & \\
\hline FLOP [Llana et al. 2012] & $\mathrm{X}$ & & & $\mathrm{X}$ & & $\mathrm{X}$ & & & & & & \\
\hline Jutge.org [Petit et al. 2012] & $\mathrm{X}$ & & $\mathrm{X}$ & & & & & & & & & \\
\hline YOJ [Sun et al. 2014] & $\mathrm{X}$ & & & $\mathrm{X}$ & & & & & & & & \\
\hline Athene [Pettit et al. 2015a] & & & & & & & & & & & & \\
\hline *Experiencia UL-FCIS [Pozenel et al. 2015] & & & & & & & & & & & & \\
\hline ViLLE [Rajala et al. 2016] & & $\mathrm{X}$ & & & $\mathrm{X}$ & & & & $\mathrm{X}$ & & & \\
\hline ProgTest [Souza et al. 2015] & $\mathrm{X}$ & & & $\mathrm{X}$ & & & & & & & & \\
\hline Test My Code [Vihavainen et al. 2013] & $\mathrm{X}$ & & & & & & & & $\mathrm{X}$ & & & \\
\hline
\end{tabular}

\section{QP2: Quais os problemas ao usar Juiz Online no ensino de CS1?}

A Tabela 6 resume os principais problemas identificados nos trabalhos lidos. Grande parte deles estão relacionados à falta de validação, de maturidade dos sistemas e à dificuldade de instalação e manipulação dos programas. Existe também a preocupação com segurança e escalabilidade que impacta na gestão da infra-estrutura. Talvez devido à falta de maturidade e à origem baseada em competições, os sistemas apresentam problemas de usabilidade. O processo de submissão de programas ainda apresenta problemas, e o feedback não é suficiente para que alunos consigam corrigir muitos dos erros.

Tabela 6. Problemas encontrados nos trabalhos

\begin{tabular}{|c|c|c|}
\hline PROBLEMA & REFERÊNCIAS & $\mathbf{N}^{0}$ TRABALHOS \\
\hline A - Falta de maturidade dos sistemas & [A1Shamsi and Elnagar 2011], [Pozenel et al. 2015] & 2 \\
\hline B - Falta de validação do sistema & $\begin{array}{c}\text { [Pettit et al. 2015b], [Llana et al. 2012], [Pettit et al. 2015a], } \\
\text { [Pozenel et al. 2015] }\end{array}$ & 4 \\
\hline $\begin{array}{l}\text { C - Dificuldade em gerenciar a } \\
\text { infra-estrutura }\end{array}$ & [Petit et al. 2012] & 1 \\
\hline D - Usabilidade & [Souza et al. 2015] & 1 \\
\hline $\begin{array}{l}\text { E - Disponibilizar publicamente a nota } \\
\text { de todos os alunos }\end{array}$ & [Georgouli and Guerreiro 2010] & 1 \\
\hline $\begin{array}{l}\text { F - Sistema contextualizado em } \\
\text { competições }\end{array}$ & [Georgouli and Guerreiro 2010], [Sun et al. 2014] & 2 \\
\hline $\begin{array}{l}\text { G - Falta da informação sobre a } \\
\text { contribuição dos sistemas para a } \\
\text { aprendizagem }\end{array}$ & $\begin{array}{l}\text { [Pettit et al. 2015b], [Georgouli and Guerreiro 2010], } \\
\text { [Wang et al. 2011] }\end{array}$ & 3 \\
\hline $\begin{array}{l}\text { H - Sistema aumentou ocorrência de } \\
\text { plágio }\end{array}$ & [Pozenel et al. 2015] & 1 \\
\hline I - Aluno não testar seus programas & [Vihavainen et al. 2013] & 1 \\
\hline
\end{tabular}

Outros problemas surgem de decisões de implementação e das implicações do uso do sistema na sala de aula, e estão relacionados à interpretação dos usuários. Alguns alunos não gostam que as notas estejam visíveis para todos e nem do ambiente de competição que o uso de sistemas de Juiz Online criam. O fato do sistema fazer a correção automática pode levar a uma programação na base de tentativa e erro e ao aumento da ocorrência de plágio. Além do mais, não parece haver aumento das notas dos alunos [Wang et al. 2011].

A Tabela 7 relaciona os sistemas de Juiz Online com os problemas identificados na Tabela 6.

\section{QP3: Quais requisitos funcionais um Juiz Online precisa atender para ser usado no ensino de CS1?}

A leitura dos trabalhos sobre CS1 e Juiz Online trouxe quatro conjuntos de requisitos funcionais que podem variar conforme o contexto de ensino. A Tabela 8 mostra a 
V Congresso Brasileiro de Informática na Educação (CBIE 2016)

Anais do XXVII Simpósio Brasileiro de Informática na Educação (SBIE 2016)

Tabela 7. Juízes Online relacionados aos problemas

\begin{tabular}{|l|c|c|c|c|c|c|c|c|c|}
\hline SISTEMA & A & B & C & D & E & F & G & H & I \\
\hline Mooshak [Georgouli and Guerreiro 2010] & & & & & X & X & X & & \\
\hline AutoLEP [Wang et al. 2011] & & & & & & & $\mathrm{X}$ & & \\
\hline eGrader [AlShamsi and Elnagar 2011] & $\mathrm{X}$ & & & & & & & & \\
\hline FLOP [Llana et al. 2012] & & $\mathrm{X}$ & & & & & & & \\
\hline Jutge.org [Petit et al. 2012] & & & $\mathrm{X}$ & & & & & & \\
\hline YOJ [Sun et al. 2014] & & & & & & $\mathrm{X}$ & & & \\
\hline Athene [Pettit et al. 2015a] & & $\mathrm{X}$ & & & & & & & \\
\hline *Experiência UL-FCIS [Pozenel et al. 2015] & $\mathrm{X}$ & $\mathrm{X}$ & & & & & & $\mathrm{X}$ & \\
\hline ViLLE [Rajala et al. 2016] & & & & & & & & & \\
\hline ProgTest [Souza et al. 2015] & & & & $\mathrm{X}$ & & & & & \\
\hline Test My Code [Vihavainen et al. 2013] & & & & & & & & & $\mathrm{X}$ \\
\hline
\end{tabular}

distribuição de trabalhos quanto a esses conjuntos.

Tabela 8. Requisitos funcionais encontrados nos trabalhos

\begin{tabular}{|c|c|c|}
\hline REQUISITO & REFERÊNCIAS & No TRABALHOS \\
\hline $\mathrm{C} 1$ - Feedback & $\begin{array}{c}\text { [Georgouli and Guerreiro 2010], [Wang et al. 2011], } \\
\text { [A1Shamsi and Elnagar 2011], [Llana et al. 2012] [Petit et al. 2012], } \\
\text { [Sun et al. 2014], [Pettit et al. 2015a], [Pozenel et al. 2015] } \\
\text { [Rajala et al. 2016], [Souza et al. 2015], [Vihavainen et al. 2013] }\end{array}$ & 11 \\
\hline $\begin{array}{l}\text { C2 - Integração do } \\
\text { Sistema com os } \\
\text { Cursos }\end{array}$ & $\begin{array}{l}\text { [Wang et al. 2011], [Llana et al. 2012], [Petit et al. 2012], } \\
\text { [Sun et al. 2014] [Pozenel et al. 2015], [Rajala et al. 2016], } \\
\text { [Souza et al. 2015], [Vihavainen et al. 2013] }\end{array}$ & 8 \\
\hline $\begin{array}{l}\text { C3 - Análise do } \\
\text { Desempenho Geral } \\
\text { dos Alunos }\end{array}$ & $\begin{array}{l}\text { [Petit et al. 2012], [A1Shamsi and Elnagar 2011], } \\
\text { [Vihavainen et al. 2013] }\end{array}$ & 3 \\
\hline $\begin{array}{l}\text { C4 - Oferecer } \\
\text { Diferentes Tipos de } \\
\text { Atividades }\end{array}$ & $\begin{array}{l}\text { [Sun et al. 2014], [Pozenel et al. 2015], [Rajala et al. 2016], } \\
\text { [Souza et al. 2015] [Vihavainen et al. 2013] }\end{array}$ & 5 \\
\hline
\end{tabular}

O Feedback é fundamental para a aprendizagem do aluno e requisito chave dos sistemas de Juiz Online. Normalmente o Feedback é o resultado de testes feitos pelo Juiz Online com o programa submetido pelo aluno [Wang et al. 2011, Llana et al. 2012, Petit et al. 2012, Sun et al. 2014, Pozenel et al. 2015, Rajala et al. 2016]. Entretanto, ele pode incluir pontuação parcial [Pettit et al. 2015a, Vihavainen et al. 2013], contribuição do professor [Georgouli and Guerreiro 2010, Sun et al. 2014, Vihavainen et al. 2013] ou de outros alunos [Sun et al. 2014]. Também pode ser escondido quando o sistema é usado em exames [Rajala et al. 2016].

Fica claro pelos artigos que existe um esforço em melhorar o tratamento de erros a partir do feedback, oferecendo uma melhor descrição dos erros de compilação e execução e da saída do programa [Rajala et al. 2016], oferecendo detalhes dos casos de teste que produziram erro [Pettit et al. 2015a], considerando aspectos de sintaxe [Wang et al. 2011] ou estruturais que se relacionam com a semântica [Wang et al. 2011, AlShamsi and Elnagar 2011], e identificando a evolução do estilo de codificação [Pettit et al. 2015a]. [Souza et al. 2015] propõe avaliar o programa do aluno com os casos de teste do professor e o programa escrito pelo professor com casos de teste do aluno, além de permitir ao professor a inclusão de dicas textuais.

Alguns trabalhos se preocuparam em integrar o sistema de Juiz Online com os cursos, o que pode significar novos requisitos. Existem estudos para integrá-los em um IDE para uso em MOOCs [Vihavainen et al. 2013], ou em sistemas de gestão de aprendizagem [Petit et al. 2012], flexibilizando a configuração, 
V Congresso Brasileiro de Informática na Educação (CBIE 2016)

Anais do XXVII Simpósio Brasileiro de Informática na Educação (SBIE 2016)

o que facilita a adaptação do sistema às necessidades do curso [Sun et al. 2014, Souza et al. 2015], inclusive com a possibilidade de professores cadastrarem novos problemas [Llana et al. 2012, Souza et al. 2015]. Além disso, os sistemas podem ser usados em exames [Wang et al. 2011, Petit et al. 2012, Rajala et al. 2016], e permitem acoplar a detecção de plágio [Sun et al. 2014, Pozenel et al. 2015]. A organização de problemas por nível de dificuldade foi considerada em [Petit et al. 2012, Rajala et al. 2016].

Problemas que exigem dos alunos sua resolução por meio da escrita de programas são os mais comuns nos Juízes Online. Porém, outros tipos de atividades foram identificadas nos trabalhos, como por exemplo problemas que possuem parte do código fonte escrito e solicitam ao aluno que resolva o restante [Sun et al. 2014, Rajala et al. 2016], o envio de casos de teste [Rajala et al. 2016, Vihavainen et al. 2013] e a criação de classes com métodos que seguem assinaturas pré-definidas [Pozenel et al. 2015]. [Pozenel et al. 2015] apresentaram problemas de CS1 envolvendo computação gráfica. Já [Rajala et al. 2016] propõem problemas dos tipos (Quiz, Code shuffling, Exercício Robô e Ligue os Itens). Existem sistemas que emitem relatórios com o desempenho de alunos [AlShamsi and Elnagar 2011, Petit et al. 2012, Vihavainen et al. 2013] ou turmas [AlShamsi and Elnagar 2011].

A Tabela 9 relaciona os sistemas de Juiz Online com os requisitos funcionais identificados que foram apresentados na Tabela 8 .

Tabela 9. Juízes Online relacionados aos requisitos funcionais

\begin{tabular}{|l|l|l|l|l|}
\hline SISTEMA & Feedback & $\begin{array}{l}\text { Integração com } \\
\text { os Cursos }\end{array}$ & $\begin{array}{l}\text { Desempenho de } \\
\text { Alunos }\end{array}$ & $\begin{array}{l}\text { Diferentes } \\
\text { Atividades }\end{array}$ \\
\hline Mooshak [Georgouli and Guerreiro 2010] & $\mathrm{X}$ & & & \\
\hline AutoLEP [Wang et al. 2011] & $\mathrm{X}$ & $\mathrm{X}$ & & \\
\hline eGrader [AlShamsi and Elnagar 2011] & $\mathrm{X}$ & & $\mathrm{X}$ & \\
\hline FLOP [Llana et al. 2012] & $\mathrm{X}$ & $\mathrm{X}$ & & \\
\hline Jutge.org [Petit et al. 2012] & $\mathrm{X}$ & $\mathrm{X}$ & $\mathrm{X}$ & $\mathrm{X}$ \\
\hline YOJ [Sun et al. 2014] & $\mathrm{X}$ & $\mathrm{X}$ & & \\
\hline Athene [Pettit et al. 2015a] & $\mathrm{X}$ & & & $\mathrm{X}$ \\
\hline${ }^{*}$ Experiência UL-FCIS [Pozenel et al. 2015] & $\mathrm{X}$ & $\mathrm{X}$ & & $\mathrm{X}$ \\
\hline ViLLE [Rajala et al. 2016] & $\mathrm{X}$ & $\mathrm{X}$ & $\mathrm{X}$ \\
\hline ProgTest [Souza et al. 2015] & $\mathrm{X}$ & $\mathrm{X}$ & $\mathrm{X}$ & $\mathrm{X}$ \\
\hline Test My Code [Vihavainen et al. 2013] & $\mathrm{X}$ & $\mathrm{X}$ & \\
\hline
\end{tabular}

QP4: Quais requisitos não-funcionais um Juiz Online precisa atender para ser usado no ensino de CS1?

Os requisitos não-funcionais que o sistema precisa atender são dependentes dos requisitos funcionais definidos. A Tabela 10 mostra a distribuição dos requisitos nãofuncionais encontrados nos trabalhos.

Tabela 10. Requisitos não-funcionais encontrados nos trabalhos

\begin{tabular}{|c|l|l|}
\hline REQUISITO & REFERÊNCIAS & N $^{\mathbf{0}}$ TRABALHOS \\
\hline Integração & [Vihavainen et al. 2013, Georgouli and Guerreiro 2010] & 2 \\
\hline Usabilidade & [Llana et al. 2012] & 1 \\
\hline Segurança & [Llana et al. 2012, Petit et al. 2012, Pozenel et al. 2015, Rajala et al. 2016] & 4 \\
\hline Escalabilidade & [Petit et al. 2012, Pozenel et al. 2015] & 2 \\
\hline Disponibilidade & [Vihavainen et al. 2013, Llana et al. 2012, Sun et al. 2014] & 3 \\
\hline
\end{tabular}

Existe um grande interesse em desenvolver sistemas de Juiz Online para serem amplamente usados na internet [Llana et al. 2012, Petit et al. 2012, Pozenel et al. 2015, 
V Congresso Brasileiro de Informática na Educação (CBIE 2016)

Anais do XXVII Simpósio Brasileiro de Informática na Educação (SBIE 2016)

Rajala et al. 2016]. Nesse contexto, alguns aspectos, principalmente de segurança, devem ser considerados, como o tratamento de programas mal-intencionados [Llana et al. 2012, Petit et al. 2012] e o uso de firewall [Rajala et al. 2016]. [Pozenel et al. 2015] propõem a execução dos programas submetidos pelos alunos como um usuário sem privilégios no linux para lidar com a segurança.

A escalabilidade foi tratada pelos trabalhos [Petit et al. 2012, Pozenel et al. 2015], e deve ser considerada quando se tem o interesse em um sistema com quantidade crescente de usuários e transações, implicando no requisito não-funcional disponibilidade [Vihavainen et al. 2013, Llana et al. 2012, Sun et al. 2014], especialmente presente quando usados em MOOCs.

A Tabela 11 relaciona os sistemas de Juiz Online com os requisitos nãofuncionais identificados. Os requisitos não-funcionais descritos são integração, usabilidade, segurança, escalabilidade, e disponibilidade. Os dados mostram que não há uma forte preocupação com esses requisitos, o que pode ser devido aos sistemas ainda não terem uma certa maturidade. Acredita-se que quando houver uma popularização maior dos sistemas no ensino, os projetos buscarão atacar mais tais questões.

Tabela 11. Juízes Online relacionados aos requisitos não-funcionais
\begin{tabular}{|l|l|l|l|l|l|}
\hline SISTEMA & Integ. & Usabil. & Seg. & Escalab. & Disponib. \\
\hline Mooshak [Georgouli and Guerreiro 2010] & $\mathrm{X}$ & & & & \\
\hline AutoLEP [Wang et al. 2011] & & & & & \\
\hline eGrader [AlShamsi and Elnagar 2011] & & & & & \\
\hline FLOP [Llana et al. 2012] & & $\mathrm{X}$ & $\mathrm{X}$ & & $\mathrm{X}$ \\
\hline Jutge.org [Petit et al. 2012] & & & $\mathrm{X}$ & $\mathrm{X}$ & \\
\hline YOJ [Sun et al. 2014] & & & & & $\mathrm{X}$ \\
\hline Athene [Pettit et al. 2015a] & & & & & \\
\hline${ }^{*}$ Experiência UL-FCIS [Pozenel et al. 2015] & & & $\mathrm{X}$ & $\mathrm{X}$ & \\
\hline ViLLE [Rajala et al. 2016] & & & $\mathrm{X}$ & & \\
\hline ProgTest [Souza et al. 2015] & & & & & \\
\hline Test My Code [Vihavainen et al. 2013] & $\mathrm{X}$ & & & & $\mathrm{X}$ \\
\hline
\end{tabular}

\section{Conclusão}

Para responder à questão Qual é a especificação mais adequada para que um juiz online atenda à disciplina de CS1? é necessário explorar diferentes configurações de requisitos de sistemas de Juiz Online nos diferentes contextos educacionais e levantar a contribuição para a aprendizagem do aluno. A variedade de requisitos funcionais e não-funcionais possíveis é grande, como apresenta esta RSL. A leitura dos artigos selecionados possibilitou classificar os requisitos funcionais em quatro grupos (feedback, integração do sistema com os cursos, análise do desempenho geral dos alunos, e oferecer diferentes tipos de atividades) e os requisitos não-funcionais (integração, usabilidade, segurança, escalabilidade, disponibilidade).

A questão da eficácia educacional das ferramentas de Juiz Online ainda é um problema em aberto. [Ramos et al. 2015] mostraram que somente 10.2\% dos artigos avaliados em seu trabalho apresentaram rigor nos resultados, trazendo a validação das ferramentas como um desafio. No entanto, esta RSL apontou diversos benefícios e problemas que podem ocorrer no uso dessas ferramentas. Essa análise é importante para saber quais são os problemas que precisam ser resolvidos e apoiar na busca dos benefícios. Aprendizagem no ritmo do aluno, auto-aprendizagem e redução da carga de trabalho do professor, 
V Congresso Brasileiro de Informática na Educação (CBIE 2016)

Anais do XXVII Simpósio Brasileiro de Informática na Educação (SBIE 2016)

são alguns dos benefícios apontados que contribuem não só em ambientes tradicionais de ensino, mas em ambientes de Educação a Distância (EAD) e em MOOC's. A liberdade de definir listas de exercício e a disponibilidade de instrumentos para acompanhar os alunos são questões importantes para o professor.

O Feedback é fundamental nos sistemas de Juiz Online. Existem projetos que visam melhorá-lo, trazendo informações sobre aspectos de sintaxe ou estruturais que se relacionam com a semântica e possibilitando identificar a evolução do estilo de codificação do aluno. Isso traz novas perspectivas para a pesquisa.

O levantamento feito aponta a importância de analisar a qualidade de ferramentas educacionais. É necessário definir padrões que possibilitem comparar as ferramentas nos diferentes contextos de ensino em uma abordagem mais formal e rigorosa. Vale observar também, que existem sistemas de Juiz Online que estão sendo produzidos e usados no Brasil com bastante popularidade, como o $B O C A^{1}$ e o URI ${ }^{2}$, que não foram tratados nesta RSL por não terem sido retornados na pesquisa realizada conforme protocolo definido.

\section{Referências}

AlShamsi, F. and Elnagar, A. (2011). An automated assessment and reporting tool for introductory java programs. In Innovations in Information Technology (IIT), 2011 International Conference on, pages 324-329. IEEE.

Byrne, P. and Lyons, G. (2001). The effect of student attributes on success in programming. In ACM SIGCSE Bulletin, volume 33, pages 49-52. ACM.

Georgouli, K. and Guerreiro, P. (2010). Incorporating an automatic judge into blended learning programming activities. In Advances in Web-Based Learning-ICWL 2010, pages 81-90. Springer.

Gomes, A., Areias, C., Henriques, J., and Mendes, A. J. (2008). Aprendizagem de programação de computadores: dificuldades e ferramentas de suporte. Revista Portuguesa de Pedagogia, 42(2).

Kitchenham, B. (2004). Procedures for performing systematic reviews. Keele, UK, Keele University, 33(2004):1-26.

Kurnia, A., Lim, A., and Cheang, B. (2001). Online judge. Computers \& Education, 36(4):299- 315.

Llana, L., Martin-Martin, E., and Pareja-Flores, C. (2012). Flop, a free laboratory of programming. In Proceedings of the 12th Koli Calling International Conference on Computing Education Research, pages 93-99. ACM.

Petit, J., Giménez, O., and Roura, S. (2012). Jutge. org: an educational programming judge. In Proceedings of the 43rd ACM technical symposium on Computer Science Education, pages 445-450. ACM.

Pettit, R., Homer, J., Gee, R., Mengel, S., and Starbuck, A. (2015a). An empirical study of iterative improvement in programming assignments. In Proceedings of the 46th ACM Technical Symposium on Computer Science Education, pages 410-415. ACM.

\footnotetext{
${ }^{1}$ BOCA: disponível em www. ime.usp.br/ cassio/boca/

${ }^{2}$ URI: disponível em www . urionlinejudge.com.br
} 
V Congresso Brasileiro de Informática na Educação (CBIE 2016)

Anais do XXVII Simpósio Brasileiro de Informática na Educação (SBIE 2016)

Pettit, R., Homer, J., Holcomb, K., Simone, N., and Mengel, S. (2015b). Are automated assessment tools helpful in programming courses? 122nd ASEE Annual Conference and Exposition. American Society for Engineering Education, 2015.

Pozenel, M., Furst, L., and Mahnicc, V. (2015). Introduction of the automated assessment of homework assignments in a university-level programming course. In Information and Communication Technology, Electronics and Microelectronics (MIPRO), 2015 38th International Convention on, pages 761-766. IEEE.

Rajala, T., Kaila, E., Lindén, R., Kurvinen, E., Lokkila, E., Laakso, M.-J., and Salakoski, T. (2016). Automatically assessed electronic exams in programming courses. In Proceedings of the Australasian Computer Science Week Multiconference, page 11. ACM.

Ramos, V., Wazlawick, R., Galimberti, M., Freitas, M., and Mariani, A. C. (2015). A comparação da realidade mundial do ensino de programação para iniciantes com a realidade nacional: Revisão sistemática da literatura em eventos brasileiros. In Anais do Simpósio Brasileiro de Informática na Educação, volume 26, page 318.

Rountree, N., Rountree, J., Robins, A., and Hannah, R. (2004). Interacting factors that predict success and failure in a cs1 course. ACM SIGCSE Bulletin, 36(4):101-104.

Souza, D. M. D., Isotani, S., and Barbosa, E. F. (2015). Teaching novice programmers using progtest. International Journal of Knowledge and Learning, 10(1):60-77.

Sun, H., Li, B., and Jiao, M. (2014). Yoj: An online judge system designed for programming courses. In Computer Science \& Education (ICCSE), 2014 9th International Conference on, pages 812-816. IEEE.

Vieira, M. A. (2016). Modelagem de Espaços Inteligentes Pessoais e Espaços Inteligentes Fixos no contexto de Cenários de Computação Ubíqua - Dissertação de Mestrado em Ciência da Computação. PhD thesis, Universidade Federal de Goiás.

Vier, J., Gluz, J., and Jaques, P. A. (2015). Empregando redes bayesianas para modelar automaticamente o conhecimento dos alunos em lógica de programação. Revista Brasileira de Informática na Educação, 23(02):45.

Vihavainen, A., Luukkainen, M., and Pärtel, M. (2013). Test my code: An automatic assessment service for the extreme apprenticeship method. In 2nd International Workshop on Evidence-based Technology Enhanced Learning, pages 109-116. Springer.

Wang, T., Su, X., Ma, P., Wang, Y., and Wang, K. (2011). Ability-training-oriented automated assessment in introductory programming course. Computers \& Education, 56(1):220-226.

Wilson, B. C. and Shrock, S. (2001). Contributing to success in an introductory computer science course: a study of twelve factors. In ACM SIGCSE Bulletin, volume 33, pages 184-188. ACM. 